\title{
A NOTE ON BOREL EQUIVALENCE RELATIONS
}

\author{
ALAIN LOUVEAU AND BOBAN VELICKOVIC
}

(Communicated by Andreas R. Blass)

\begin{abstract}
We prove that the class of Borel equivalence relations, under the order of Borel reducibility, is a very complicated structure, by embedding into it the partial order of inclusion modulo finite sets on $\mathscr{P}(\omega)$.
\end{abstract}

Given Borel equivalence relations $E$ and $F$ on Polish spaces $X$ and $Y$ respectively, we say that $E$ is reducible to $F$ and write $E \leq F$ if there is a Borel function $f: X \rightarrow Y$ such that, for every $x$ and $y$ in $X, x E y$ iff $f(x) F f(y)$. Let $\mathscr{E}$ be the class of all Borel equivalence relations. The question of which partial orderings can be embedded into the structure $(\mathscr{E}, \leq)$ has been studied by several authors. For a comprehensive survey see [Ke]. Just [Ju] has proved that this structure contains arbitrarily large finite antichains, and Woodin [Wo] gave an ingenious construction of a perfect antichain as well as an infinite descending chain using metamathematical means. In this note we show by completely elementary means that $(\mathscr{E}, \leq)$ is a very rich structure. We prove the following, where $\subseteq_{*}$ denotes inclusion modulo finite sets.

Theorem 1. The structure $\left(\mathscr{P}(\omega), \subseteq_{*}\right)$ can be embedded into $(\mathscr{E}, \leq)$.

Corollary 1. Every partial ordering of size $\leq \aleph_{1}$ embeds into $(\mathscr{E}, \leq)$.

Remark. It is relatively consistent with ZFC that $2^{\aleph_{0}}$ is large and $\left(\omega_{2}, \leq\right)$ does not embed into $(\mathscr{E}, \leq)$. To see this start with a model of $\mathrm{GCH}$ and add a large number of Cohen reals. The proof is identical to the proof that in this model $\mathscr{P}(\omega)$ does not contain a chain of length $\omega_{2}$ under $\subseteq_{*}$ and uses homogeneity of Cohen forcing and the fact that reducibility between Borel equivalence relations is $\Sigma_{2}^{1}$ and hence absolute.

Proof of Theorem 1. Note that if $\mathscr{J}$ is a Borel ideal in $\mathscr{P}(\omega)$ then we can define a Borel equivalence relation $E$ on $\mathscr{P}(\omega)$

$$
X E Y \text { if and only if } X \Delta Y \in \mathscr{I} \text {. }
$$

Our idea is to associate to each subset $S$ of $\omega$ a Borel ideal $\mathscr{I}_{S}$ such that the map which to each $S$ assigns the associated equivalence relation $E_{S}$ is the required embedding. In fact our embedding will have stronger properties than claimed in Theorem 1. When $S \subseteq_{*} T$ there will be a continuous reduction from $E_{S}$ to $E_{T}$, and when $S \nsubseteq_{*} T$ there will be no reduction from $E_{S}$ to

Received by the editors March 26, 1992 and, in revised form, May 8, 1992.

1991 Mathematics Subject Classification. Primary 04A15. 
$E_{T}$ which is even Baire measurable. Thus, for example, in the context of AD there is such an embedding with respect to arbitrary reductions between Borel equivalence relations. In addition, the equivalence relations we construct will be rather simple in complexity, i.e., $\Pi_{3}^{0}$ although it is still possible that there is such an embedding into $\Sigma_{2}^{0}$ equivalence relations.

To commence fix two increasing sequences of natural numbers $\left(a_{n}\right)_{n<\omega}$ and $\left(b_{n}\right)_{n<\omega}$ such that for every $n<\omega$

$$
a_{n+1} \geq 2^{n+1}\left(a_{n}+2\right) \text { and } b_{n+1} \geq 2^{a_{n+1}+(n+1) \cdot b_{n}} .
$$

Define inductively an increasing sequence of integers $\left(m_{n}\right)_{n<\omega}$ by $m_{0}=0$ and $m_{n+1}=m_{n}+b_{n}$, and let $I_{n}$ denote the interval [ $\left.m_{n}, m_{n+1}\right)$. For a subset $S$ of $\omega$ let $I_{S}=\bigcup_{n \in S} I_{n}$. To each subset $X$ of $\omega$ we associate a sequence $(X(n))_{n<\omega}$ of finite sets where $X(n)=X \cap I_{n}$. Say that a subset $G$ of $\omega$ is a graph if $\operatorname{card}\left(G \cap I_{k}\right) \leq 1$ for every $k$. For every $n$ we define an $n$-norm on $\mathscr{P}(\omega)$ as

$$
\|X\|_{n}=\frac{\log (\operatorname{card} X(n)+1)}{a_{n}} .
$$

The main property of the $n$-norm which will be used in the proof is the following inequality which is easily verified. For any sets $X_{0}, \ldots, X_{N-1}$

$$
\left\|\bigcup_{i<N} X_{i}\right\|_{n} \leq \sup _{i<N}\left\|X_{i}\right\|_{n}+\frac{\log N}{a_{n}} .
$$

To each infinite subset $S$ of $\omega$ we associate an ideal $\mathscr{I}_{S}$ defined by

$$
\mathscr{I}_{S}=\left\{X \subseteq \omega: \lim _{n \in S, n \rightarrow \infty}\|X\|_{n}=0\right\} .
$$

Note that $\mathscr{I}_{S}$ is a Polish group under symmetric difference. (Recall that a Borel subgroup of a Polish group is said to be Polish if there is a group topology on it which makes it Polish and has the same Borel sets as the original topology. This topology is necessarily unique.) Define as above the equivalence relation $E_{S}$ on $\mathscr{P}(\omega)$ by saying $X E_{S} Y$ if and only if $X \Delta Y \in \mathscr{I}_{S}$. Clearly $E_{S}$ is a $\Pi_{3}^{0}$ equivalence relation. We shall show that the map to which each $S$ associates $E_{S}$ is the required embedding. It is quite easy to see that if $S \subseteq_{*} T$ then the function to which each set $X$ associates $X \cap I_{S}$ is a continuous reduction of $E_{S}$ to $E_{T}$. Thus the theorem will be proved once we establish the following.

Lemma 1. If $S$ is not almost contained in $T$ then there is no Baire measurable function which reduces $E_{S}$ to $E_{T}$.

Proof. Notice that it suffices to prove the lemma for disjoint $S$ and $T$ since otherwise we can replace $S$ by $S \backslash T$. We first show that it suffices to prove that there are no continuous reductions from $E_{S}$ to $E_{T}$.

Lemma 2. If there is a Baire measurable reduction from $E_{S}$ to $E_{T}$ then there is an infinite subset $S^{*}$ of $S$ and a continuous reduction from $E_{S^{*}}$ to $E_{T}$.

Proof. Let $f$ be a Baire measurable reduction from $E_{S}$ to $E_{T}$, and fix a dense $G_{\delta}$ subset $W$ of $\mathscr{P}(\omega)$ such that $f$ is continuous on $W$. Let $W=\bigcap W_{n}$ where each $W_{n}$ is dense open in $\mathscr{P}(\omega)$. We inductively build an increasing sequence $\left(i_{n}\right)_{n<\omega}$ of elements of $S$ and sets $\left(Z_{n}\right)_{n<\omega}$ where $Z_{n}$ is a subset of $\left[m_{i_{n}+1}, m_{i_{n+1}}\right)$ such that for any subset $X$ of $\omega$ if $X \cap\left[m_{i_{n}+1}, m_{i_{n+1}}\right)=Z_{n}$ 
then $X \in W_{n}$. Then set $S^{*}=\left\{i_{n}: n<\omega\right\}$ and $Z=\bigcup_{n<\omega} Z_{n}$. Note that $Z$ is disjoint from $I_{S^{*}}$. Finally define a function $f^{*}: \mathscr{P}(\omega) \rightarrow \mathscr{P}(\omega)$ by setting

$$
f^{*}(X)=f\left(\left(X \cap I_{S^{*}}\right) \cup Z\right) \text {. }
$$

Then it is easily seen that $f^{*}$ is a continuous reduction of $E_{S^{*}}$ to $E_{T}$.

To simplify notation let us now assume that we have a continuous function $f: \mathscr{P}(\omega) \rightarrow \mathscr{P}(\omega)$ such that, for every $X, Y \in \mathscr{P}(\omega), X \Delta Y \in \mathscr{I}_{S}$ if and only if $f(X) \Delta f(Y) \in \mathscr{I}_{T}$. We shall derive a contradiction. Notice that we may assume that the range of $f$ is contained in $\mathscr{P}\left(I_{T}\right)$.

We inductively define increasing sequences $\left(i_{n}\right)_{n<\omega}$ and $\left(j_{n}\right)_{n<\omega}$ of members of $S$ and $T$ respectively and sequences of finite sets $\left(A_{n}\right)_{n<\omega},\left(B_{n}\right)_{n<\omega}$, $\left(G_{n}\right)_{n<\omega},\left(A_{n}^{*}\right)_{n<\omega}$, and $\left(B_{n}^{*}\right)_{n<\omega}$ such that: $A_{n}, B_{n}$, and $G_{n}$ are subsets of $m_{i_{n}} ; A_{n}^{*}$ and $B_{n}^{*}$ are subsets of $m_{j_{n}}$; and $G_{n}$ is a graph. We require that $A_{n+1} \cap m_{i_{n}}=A_{n}, B_{n+1} \cap m_{i_{n}}=B_{n}, G_{n+1} \cap m_{i_{n}}=G_{n}$ and that the following conditions be satisfied:

1. For all $X, G \subseteq \omega \backslash m_{i_{n}}$ with $G$ a graph, the following hold:

(a) $f\left(A_{n} \cup X\right) \cap m_{j_{n}}=A_{n}^{*}$ and $f\left(B_{n} \cup X\right) \cap m_{j_{n}}=B_{n}^{*}$.

(b) For all $p \geq j_{n},\left\|f\left(A_{n} \cup X\right) \Delta f\left(B_{n} \cup X\right)\right\|_{p} \leq 2^{-(n+1)}$.

(c) For all $p \geq j_{n},\left\|f\left(A_{n} \cup X\right) \Delta f\left(\left(A_{n} \cup X\right) \Delta\left(G_{n} \cup G\right)\right)\right\|_{p} \leq 2^{-(n+1)}$.

2. (a) $\left\|A_{n+1} \Delta B_{n+1}\right\|_{i_{n}} \geq 1$.

(b) For $j_{n} \leq p<j_{n+1},\left\|A_{n+1}^{*} \Delta B_{n+1}^{*}\right\|_{p} \leq 2^{-n}$.

In the end define $A=\bigcup_{n<\omega} A_{n}$ and $B=\bigcup_{n<\omega} B_{n}$. Then $f(A)=\bigcup_{n<\omega} A_{n}^{*}$ and $f(B)=\bigcup_{n<\omega} B_{n}^{*}$. It follows from 2(a) that $A \not_{S} B$ and from 2(b) that $f(A) E_{T} f(B)$. Thus to finish the proof it suffices to do the inductive step of the construction.

Suppose now the construction has been carried out through stage $n$. We do it at stage $n+1$. Pick a family $\mathscr{F}$ of disjoint subsets of $I_{i_{n}}$, each of cardinality $2^{a_{i_{n}}-1}$ such that card $\mathscr{F}=2^{m_{i_{n}}}+1$. Fix some $X_{0} \subseteq \omega \backslash m_{i_{n}+1}$. By the pigeon-hole principle there are distinct $A$ and $B$ in $\mathscr{F}$ such that

$$
f\left(A_{n} \cup A \cup X_{0}\right) \uparrow m_{i_{n}}=f\left(A_{n} \cup B \cup X_{0}\right) \uparrow m_{i_{n}} .
$$

By continuity there is some $i^{\prime}>i_{n}+1$ such that, for any $X \subseteq \omega \backslash m_{i_{n}+1}$, if $X \cap\left[m_{i_{n}+1}, m_{i^{\prime}}\right)=X_{0} \cap\left[m_{i_{n}+1}, m_{i^{\prime}}\right)$ then the above equality is true with $X$ instead of $X_{0}$ and the value is fixed. Let $X_{0}^{*}=X_{0} \cap\left[m_{i_{n}+1}, m_{i^{\prime}}\right)$.

Claim 1. For every $X \subseteq \omega \backslash m_{i^{\prime}}$ and $p \geq j_{n}$

$$
\left\|f\left(A_{n} \cup A \cup X_{0}^{*} \cup X\right) \Delta f\left(B_{n} \cup B \cup X_{0}^{*} \cup X\right)\right\|_{p} \leq 2^{-n} .
$$

Proof. The main point is that $i_{n} \notin T$. So the case $p=i_{n}$ is trivial, since the range of $f$ is contained in $\mathscr{P}\left(I_{T}\right)$. Thus we have two cases to consider.

Case 1. $p<i_{n}$. First of all from the choice of $A$ and $B$ it follows that $f\left(A_{n} \cup A \cup X_{0}^{*} \cup X\right) \uparrow m_{i_{n}}=f\left(A_{n} \cup B \cup X_{0}^{*} \cup X\right) \uparrow m_{i_{n}}$. Also by $1(\mathrm{~b})$

$$
\left\|f\left(A_{n} \cup B \cup X_{0}^{*} \cup X\right) \Delta f\left(B_{n} \cup B \cup X_{0}^{*} \cup X\right)\right\|_{p} \leq 2^{-(n+1)} .
$$

Combining these two we obtain the claim in this case.

Case 2. $p>i_{n}$. Enumerate the set $A \cup B$ as $\left\{t_{0}, \ldots, t_{2^{a_{n-1}}}\right\}$, and define $A^{(k)}=A \Delta\left\{t_{i}: i<k\right\}$. Note that $A^{(0)}=A$ and $A^{\left(2^{a_{i_{n}}}\right)}=B$. Fix any $X \subseteq \omega \backslash m_{i^{\prime}}$ and a graph $G \subseteq \omega \backslash m_{i_{n}+1}$. For each $k$ consider the set

$$
U_{k}=f\left(A_{n} \cup A^{(k)} \cup X_{0}^{*} \cup X\right) \Delta f\left(\left(A_{n} \cup A^{(k)} \cup X_{0}^{*} \cup X\right) \Delta\left(G_{n} \cup\left\{t_{k}\right\} \cup G\right)\right) \text {. }
$$


Then by $1(\mathrm{c})\left\|U_{k}\right\|_{p} \leq 2^{-(n+1)}$. On the other hand $1(\mathrm{~b})$ implies that

$$
\left\|f\left(A_{n} \cup B \cup X_{0}^{*} \cup X\right) \Delta f\left(B_{n} \cup B \cup X_{0}^{*} \cup X\right)\right\|_{p} \leq 2^{-(n+1)} .
$$

Now since $f\left(A_{n} \cup A \cup X_{0}^{*} \cup X\right) \Delta f\left(B_{n} \cup B \cup X_{0}^{*} \cup X\right)$ is contained in the union of $f\left(A_{n} \cup B \cup X_{0}^{*} \cup X\right) \Delta f\left(B_{n} \cup B \cup X_{0}^{*} \cup X\right)$ and $\cup_{k \leq 2^{a_{i n}}} U_{k}$, using inequality (1) we obtain that

$$
\left\|f\left(A_{n} \cup A \cup X_{0}^{*} \cup X\right) \Delta f\left(B_{n} \cup B \cup X_{0}^{*} \cup X\right)\right\|_{p} \leq 2^{-(n+1)}+\frac{a_{i_{n}}+2}{a_{p}} \leq 2^{-n} .
$$

This proves Claim 1. . .]

Now let $A_{n}^{\prime}=A_{n} \cup A \cup X_{0}^{*}$ and $B_{n}^{\prime}=B_{n} \cup B \cup X_{0}^{*}$. To define $A_{n+1}$ and $B_{n+1}$ we shall end-extend $A_{n}^{\prime}$ and $B_{n}^{\prime}$ by the same set. Since $\left\|A_{n}^{\prime} \Delta B_{n}^{\prime}\right\|_{i_{n}}=1$ and by Claim 1 we have guaranteed condition 2(b) no matter which common extension we pick, we only have to worry about satisfying conditions $1(a)-(c)$.

Claim 2. There is $j>i^{\prime}$ and $Z \subseteq\left[m_{i^{\prime}}, m_{j}\right)$ such that for every $X \subseteq \omega \backslash m_{j}$ and for every $p \geq j$

$$
\left\|f\left(A_{n}^{\prime} \cup Z \cup X\right) \Delta f\left(B_{n}^{\prime} \cup Z \cup X\right)\right\|_{p} \leq 2^{-(n+2)} .
$$

Proof. Otherwise we can build strictly increasing sequences of integers $\left(s_{k}\right)_{k<\omega}$ and $\left(t_{k}\right)_{k<\omega}$ and a sequence of sets $\left(Z_{k}\right)_{k<\omega}$ such that $s_{0}=i^{\prime}, Z_{0}=\varnothing$, and, for every $k, Z_{k} \subseteq\left[m_{i^{\prime}}, m_{s_{k}}\right), Z_{k+1} \cap m_{s_{k}}=Z_{k}$, and for every $X \subseteq \omega \backslash m_{s_{k}}$

$$
\left\|f\left(A_{n}^{\prime} \cup Z_{k} \cup X\right) \Delta f\left(B_{n}^{\prime} \cup Z_{k} \cup X\right)\right\|_{t_{k}} \geq 2^{-(n+2)} .
$$

Note that since the range of $f$ is contained in $\mathscr{P}\left(I_{T}\right)$ each $t_{k}$ is in $T$. In the end define $X=A_{n}^{\prime} \cup \bigcup_{k<\omega} Z_{k}, Y=B_{n}^{\prime} \cup \bigcup_{k<\omega} Z_{k}$. It follows that $X \Delta Y=$ $A_{n}^{\prime} \Delta B_{n}^{\prime} \in \mathscr{I}_{S}$, but $f(X) \Delta f(Y) \notin \mathscr{I}_{T}$. Hence $f$ is not a reduction of $E_{S}$ to $E_{T}$.

Now fix $Z$ and $j$ as in Claim 2, and define $A_{n}^{\prime \prime}=A_{n}^{\prime} \cup Z$ and $B_{n}^{\prime \prime}=B_{n}^{\prime} \cup Z$. $A_{n+1}$ and $B_{n+1}$ will be built as end-extensions of $A_{n}^{\prime \prime}$ and $B_{n}^{\prime \prime}$ respectively, and thus by Claim 2 condition $1(\mathrm{~b})$ is guaranteed, provided that we choose $j_{n+1} \geq j$. We now take care of condition 1 (c).

Claim 3. There is $j^{\prime}>j$ and $W, H \subseteq\left[m_{j}, m_{j^{\prime}}\right)$ such that $H$ is a graph and such that for every $X, G \subseteq \omega \backslash m_{j^{\prime}}$ with $G$ a graph and every $p \geq j^{\prime}$

$$
\left\|f\left(A_{n}^{\prime \prime} \cup W \cup X\right) \Delta f\left(\left(A_{n}^{\prime \prime} \cup W \cup X\right) \Delta\left(G_{n} \cup H \cup G\right)\right)\right\|_{p} \leq 2^{-(n+2)} .
$$

Proof. Otherwise as in Claim 2 we can build strictly increasing sequences of integers $\left(s_{k}\right)_{k<\omega}$ and $\left(t_{k}\right)_{k<\omega}$ and sequences of sets $\left(W_{k}\right)_{k<\omega}$ and $\left(H_{k}\right)_{k<\omega}$ such that $s_{0}=j^{\prime}, W_{0}=H_{0}=\varnothing$, and, for every $k, s_{k}<t_{k}<s_{k+1}, W_{k}$, $H_{k} \subseteq\left[m_{j}, m_{s_{k}}\right)$ with $H_{k}$ a graph, $W_{k+1} \cap m_{s_{k}}=W_{k}, H_{k+1} \cap m_{s_{k}}=H_{k}$, and for every $X, G \subseteq \omega \backslash m_{s_{k}}$ with $G$ a graph

$$
\left\|f\left(A_{n}^{\prime \prime} \cup W_{k} \cup X\right) \Delta f\left(\left(A_{n}^{\prime \prime} \cup W_{k} \cup X\right) \Delta\left(G_{n} \cup H_{k} \cup G\right)\right)\right\|_{t_{k}} \geq 2^{-(n+2)} .
$$

As before it follows that each $t_{k}$ is in $T$. Finally let $X=A_{n}^{\prime \prime} \cup \bigcup_{k<\omega} W_{k}$, $G=G_{n} \cup \bigcup_{k<\omega} H_{k}$, and $Y=X \Delta G$. Then $X \Delta Y=G$ is a graph and hence in $\mathscr{I}_{S}$, but $f(X) \Delta f(Y) \notin \mathscr{I}_{T}$. Contradiction. 
Fix $W, H$, and $j^{\prime}$ as in Claim 3. Set $j_{n+1}=j^{\prime}$, and define $A_{n}^{\prime \prime \prime}=A_{n}^{\prime \prime} \cup$ $W$ and $B_{n}^{\prime \prime \prime}=B_{n}^{\prime \prime} \cup W$. Now by continuity of $f$ find $i^{*} \geq j_{n+1}$ and $U \subseteq$ $\left[m_{j_{n+1}}, m_{i^{*}}\right)$ such that for some $A_{n+1}^{*}, B_{n+1}^{*} \subseteq m_{j_{n+1}}$ for every $X \subseteq \omega \backslash m_{i^{*}}$

$$
f\left(A_{n}^{\prime \prime \prime} \cup U \cup X\right) \cap m_{j_{n+1}}=A_{n+1}^{*} \quad \text { and } \quad f\left(B_{n}^{\prime \prime \prime} \cup U \cup X\right) \cap m_{j_{n+1}}=B_{n+1}^{*} \text {. }
$$

Then set $i_{n+1}=i^{*}$, and define $A_{n+1}=A_{n}^{\prime \prime \prime} \cup U, B_{n+1}=B_{n}^{\prime \prime \prime} \cup U$, and $G_{n+1}=$ $G_{n} \cup H$. It follows that conditions $1(\mathrm{a})$ and $1(\mathrm{c})$ are satisfied. This finishes the proofs of Lemma 1 and Theorem 1.

\section{REFERENCES}

[Ju] W. Just, More incomparable ideals, manuscript.

[Ke] A. Kechris, Lecture notes on equivalence relations, manuscript.

[Wo] H. Woodin, personal communication.

Equipe d'Analyse, Université Paris Vi, 4 Place Jussieu, 75252 Paris Cedex 5, France E-mail address: louveau@frunip62.bitnet

Department of Mathematics, York University, North York, Ontario, Canada 